\title{
Performance Analysis of Communication System with Fluctuating Beckmann Fading
}

\author{
Zakir Hussain, Asim ur Rehman Khan, Haider Mehdi and Aamir Ali
}

\begin{abstract}
In this paper, performance of device-to-device (D2D) communication system over Fluctuating Beckmann (FB) fading channels is analyzed. FB fading model is a novel generalized fading model that unifies various fading models such as Rayleigh, Nakagami, one-sided Gaussian, Rician, Rician shadowed, $\kappa-\mu$, $\kappa-\mu$ shadowed, $\eta-\mu$ and Beckmann. The considered D2D system is assumed to be affected by various FB faded co-channel interferers. Using the characteristic function $(\mathrm{CF})$ approach outage probability and success probability expressions are given. These expressions are functions of D2D and interference path-loss exponents, distance between the D2D devices, distances between interferers and the D2D receiver and, interference and D2D fading channel conditions. Maximum ratio combining (MRC) and selection combining (SC) based diversity schemes are considered to mitigate channel fading effects. D2D communication system under various conditions of channel fading and interference is numerically analyzed and discussed.
\end{abstract}

Index Terms - Co-channel Interference, Device-to-Device, Fluctuating Beckmann, Outage Probability, Success Probability

\section{INTRODUCTION}

An explosive growth in high data rate demand on cellular communication systems is expected in near future. This demand is due to the popularity of online gaming, HD video streaming and other social media services. Device-to-device (D2D) communication system has emerged as one of the promising technologies to overcome this problem [1-2]. It is considered to be one of the dynamic techniques of the 5 th generation $(5 \mathrm{G})$ cellular communication standard. D2D communication is defined as the direct communication of devices with each other, when are in proximity, without involvement of base station (BS). D2D system can enhance the data rate, spectrum utilization and, the energy efficiency of the user devices and the networks [3-4]. Despite many advantages, D2D communication brings some challenges as well. Due to insufficient wireless channel bandwidth and the loss of coordination between wireless devices, co-channel interference $(\mathrm{CCI})$ problem arises [5]. Therefore, effects of CCI should be considered for the analysis of D2D systems. In this paper, performance of the D2D communication system is analyzed with the help of outage probability and success probability. Outage probability performance of D2D communication system using stochastic geometry is studied by authors in [6]. Authors in [6], have not considered any diversity scheme for the system. In [7], authors studied outage performance of D2D system under optimal spectrum allocation strategy. Authors has discussed resource sharing method for user in the system. Authors in [8], discussed outage probability of D2D communication in a cellular network from a general threshold-based perspective. Success probability of D2D communication system over Rician fading channel under the distributed random-access control scheme is analyzed by authors in [9].

Outage and success performance analysis of D2D system in the presence of CCI is the aim of this work. The co-channel interference signals are considered to originate from any wireless device with which the system has lost coordination. The channel for the D2D and CCI signals are assumed to follow Fluctuating Beckmann (FB) distribution. FB fading model is a generalized fading model which includes many fading models as special case [10]. The one-sided Gaussian, Rayleigh, Nakagami, $\eta-\mu, \kappa-\mu, \kappa-\mu$ shadowed, Rician, Rician Shadowed and the Beckmann distribution are the special cases of FB model [10]. The $\kappa-\mu$ shadowed fading model [11] manages to capture propagation conditions like clustering and LoS fluctuation. However, it fails when there is power imbalance in the LoS and NLoS components. Fluctuating Beckmann (FB) fading model which is an extended $\kappa-\mu$ shadowed model is introduced in [10]. FB model effectively captures such scenarios. FB model is a generalization of Beckmann fading model by considering the effects of line-of-sight (LoS) fluctuation and clustering. It also takes into account the effects of power imbalance in the LoS and non-LoS components [10]. CCI effects on the performance of D2D communication system is analyzed. Use of generalized FB model has enabled us to present analytical expressions that can be used to analyze various fading conditions. The SC and MRC based diversity schemes are also considered to combat fading effects. Path loss conditions are also included in the analysis. Outage and success probability expressions for the non-identically distributed D2D and CCI signals are presented. Numerical analysis under various channel and CCI conditions are presented and discussed. Numerical results from these expressions are obtained with the help of MATLAB. The rest of paper is structured as follows: the system model is discussed in Section II. Also analytical expressions for outage probability and success probability are presented in Section II. In Section III, numerical results are presented and discussed. Finally, paper is concluded in Section IV. 
Performance Analysis of Communication System

with Fluctuating Beckmann Fading

\section{SySTEM MODEL}

A device-to-device (D2D) communication system in an interference limited environment is considered here. The system model is illustrated in Fig. 1. There are $N$ co-channel interferers that are affecting the D2D communication system. The D2D signals and co-channel interference (CCI) signals are considered to be independent and non-identically distributed. The co-channel interferers are assumed to be at different distances from the receiver of the D2D pair. The channels for D2D and CCI signals are assumed to be Fluctuating Beckmann (FB) distributed. Path-loss is a significant factor in performance degradation of any communication system. In this paper, a simplified path-loss model is considered [12]. To reduce the effects of fading maximal ratio combining (MRC) and selection combining (SC) based diversity techniques with $D$ branches are considered in the system.

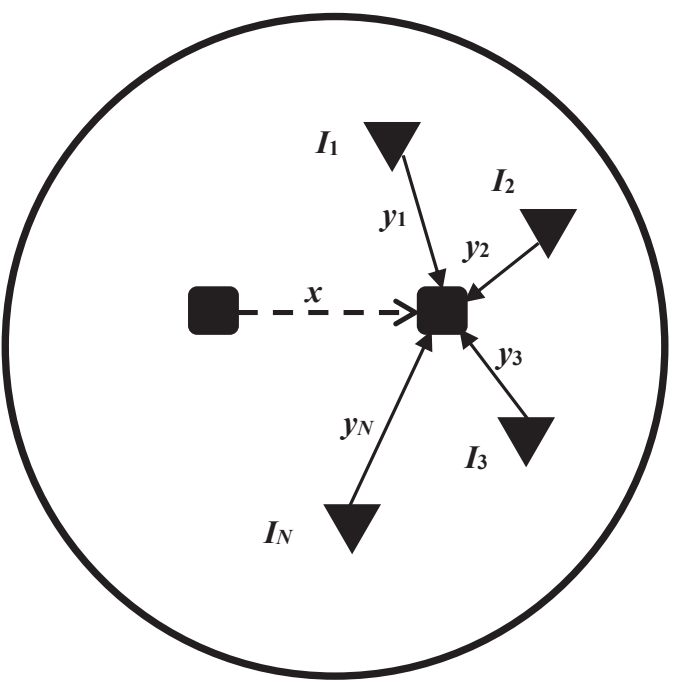

Fig. 1. System Model

D2D device

Desired D2D Signal

Distance between D2D devices

$n$-th Co-channel Interferer

Co-channel Interference Signal

Distance between the $n$-th Co-channel Interferer and

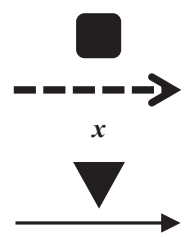

the D2D Receiver

\section{A. Selection Combining (SC) Scheme}

The signal-to-interference ratio (SIR) at the $d$-th diversity branch of the selection combining (SC) based D2D system is

$$
\frac{S_{S-S C, d}}{S_{I}}=\frac{P_{1}\left(\frac{x_{0}{ }^{u-2}}{x^{u}}\right) h_{d}}{\sum_{n=1}^{N} P_{I, n}\left(\frac{y_{0, n}^{v_{n}-2}}{y_{n}^{v_{n}}}\right) \beta_{n}}
$$

where $S_{S-S C, d}$ is the received power at the $d$-th diversity branch of $\mathrm{D} 2 \mathrm{D}$ receiver, $P_{1}$ is the power of the D2D signal, $x$ is the distance between desired D2D devices, $u$ is path-loss exponent for the D2D signal, $x_{0}$ is the reference distance and $h_{d}$ is an independent FB fading variable in the $d$-th diversity branch. Similarly, $S_{I}$ is the received power of CCI signals, $P_{I, n}$ is the power of the $n$-th CCI signal which is originated from a device at a distance $y_{n}$ from the D2D receiver, $y_{0, n}$ is the reference distance, $v_{n}$ is the path-loss exponent of the $n$-th co-channel interferer and $\beta_{n}$ is an independent FB fading variable of the $n$ th CCI signal. The outage probability is defined as the probability that the instantaneous SIR of the communication system falls below a predefined threshold $R$. The outage probability for the D2D system is

$P_{\text {out }, S C}=\operatorname{Pr}\left(R S_{I}>S_{S-S C, M A X}\right)$

where $S_{S-S C, M A X}=\max _{d=1, \ldots, D}\left(S_{S-S C, d}\right)$ and $\operatorname{Pr}($.$) is probability.$

Based on (2), a decision variable $\partial$ is defined as

$\partial=R S_{I}-S_{S, S C, M A X}$

For a successful reception, the value of $\partial$ must be less than zero, otherwise outage will occur. Mathematically,

$\partial \begin{cases}>0 & \text { Outage } \\ \leq 0 & \text { Successful Transmission }\end{cases}$

In this study, a characteristic function (CF) based approach is used for the outage analysis. The $\mathrm{CF}$ expression for the decision variable is obtained with the help of [10] and is given as,

$\phi_{\partial}(\omega)=\frac{\left[1+\frac{1}{m_{d}}\left(\frac{j \omega C_{d}}{G_{d}+j \omega E_{d}}+\frac{j \omega F_{d}}{G_{d}+j \omega H_{d}}\right)\right]^{-m_{d}}}{\left[\left(1+j \omega A_{d}\right)\left(1+j \omega B_{d}\right)\right]^{\frac{\mu_{d}}{2}}}$

$\times \prod_{n=1}^{N}\left[\frac{\left[1-\frac{1}{m_{n}}\left(\frac{j \omega C_{n}}{G_{n}-j \omega E_{n}}+\frac{j \omega F_{n}}{G_{n}-j \omega H_{n}}\right)\right]^{-m_{n}}}{\left[\left(1-j \omega A_{n}\right)\left(1-j \omega B_{n}\right)\right]^{\frac{\mu_{n}}{2}}}\right]$

where $A_{d}=\frac{2 \eta_{d} \Omega_{d} \delta_{d}}{\mu_{d}\left(1+\eta_{d}\right)\left(1+\kappa_{d}\right)}, B_{d}=\frac{2 \Omega_{d} \delta_{d}}{\mu_{d}\left(1+\eta_{d}\right)\left(1+\kappa_{d}\right)}$,

$C_{d}=\mu_{d} \kappa_{d}\left(\frac{\zeta_{d}{ }^{2}}{1+\zeta_{d}^{2}}\right)\left(1+\eta_{d}\right) \Omega_{d} \delta_{d}, \quad E_{d}=2 \eta_{d} \Omega_{d} \delta_{d}$, 
$G_{d}=\left(1+\eta_{d}\right)\left(1+\kappa_{d}\right) \mu_{d}$, $H_{d}=2 \Omega_{d} \delta_{d}$,

$F_{d}=\mu_{d} \kappa_{d}\left(\frac{1}{1+\zeta_{d}^{2}}\right)\left(1+\eta_{d}\right) \Omega_{d} \delta_{d}$ and $A_{n}=\frac{2 \eta_{n} \Omega_{n} \delta_{n}}{\mu_{n}\left(1+\eta_{n}\right)\left(1+\kappa_{n}\right)}$, $B_{n}=\frac{2 \Omega_{n} \delta_{n}}{\mu_{n}\left(1+\eta_{n}\right)\left(1+\kappa_{n}\right)}, \quad C_{n}=\mu_{n} \kappa_{n}\left(\frac{\zeta_{n}{ }^{2}}{1+\zeta_{n}{ }^{2}}\right)\left(1+\eta_{n}\right) \Omega_{n} \delta_{n}$, $E_{n}=2 \eta_{n} \Omega_{n} \delta_{n}, F_{n}=\mu_{n} \kappa_{n}\left(\frac{1}{1+\zeta_{n}^{2}}\right)\left(1+\eta_{n}\right) \Omega_{n} \delta_{n}, \quad H_{n}=2 \Omega_{n} \delta_{n}$, $G_{n}=\left(1+\eta_{n}\right)\left(1+\kappa_{n}\right) \mu_{n}$, where $\mu_{d}$ and $\mu_{n}$ represent number of clusters of the $d$-th branch D2D signal and $n$-th CCI signal, respectively. $\kappa_{d}$ and $\kappa_{n}$ are related to the strength of line-ofsight (LoS) component, $\eta_{d}$ and $\eta_{n}$ are the in-phase/quadrature power imbalance in the non-LoS components of the $d$-th branch D2D signal and $n$-th CCI signal, respectively. $\zeta_{d}^{2}$ and $\zeta_{n}^{2}$ are the in-phase/quadrature power imbalance in the LoS components, $m_{d}$ and $m_{n}$ accounts the fluctuation in LoS components, and, $\Omega_{d}$ and $\Omega_{n}$ are average power of the $d$-th branch D2D signal and $n$-th CCI signal, respectively. Moreover, $\delta_{d}=P_{1}\left(\frac{x_{0}^{u-2}}{x^{u}}\right)$ and $\delta_{n}=R P_{I, n}\left(\frac{y_{0, n}^{v_{n}-2}}{x^{v_{n}}}\right)$. Outage probability of the D2D system is obtained by using the identity, Pout $=\frac{1}{2}+\frac{1}{\pi} \int_{0}^{\infty} \frac{\operatorname{Im}\left(\phi_{\partial}(\omega)\right)}{\omega} d \omega$, where $\operatorname{Im}$ (.) gives the imaginary part. The outage probability for the SC diversity based D2D system is

$$
P_{\text {out }, S C}=\prod_{d=1}^{D}\left[\frac{1}{2}+\frac{1}{\pi} \int_{0}^{\infty} \frac{\operatorname{Im}\left(\phi_{\partial}(\omega)\right)}{\omega} d \omega\right]
$$

The outage expression in (6) is for the independent but nonidentically distributed D2D and CCI signals. The outage probability for independent and identically distributed case is

$$
P_{o u t, S C}=\left[\frac{1}{2}+\frac{1}{\pi} \int_{0}^{\infty} \frac{\operatorname{Im}\left(\phi_{\partial}(\omega)\right)}{\omega} d \omega\right]^{D}
$$

Success probability is defined as the probability that the SIR of the communication system remains above a predefined threshold $R$. The expression for the success probability of SC diversity based D2D system is

$$
P_{S, S C}=1-\prod_{d=1}^{D}\left[\frac{1}{2}+\frac{1}{\pi} \int_{0}^{\infty} \frac{\operatorname{Im}\left(\phi_{\partial}(\omega)\right)}{\omega} d \omega\right]
$$

The success probability expression presented in (8) is for the independent but non-identically distributed D2D and CCI signals. The success probability expression for the independent and identically distributed case is

$$
P_{S, S C}=1-\left[\frac{1}{2}+\frac{1}{\pi} \int_{0}^{\infty} \frac{\operatorname{Im}\left(\phi_{\partial}(\omega)\right)}{\omega} d \omega\right]^{D}
$$

\section{B. Maximal Ratio Combining (MRC) Scheme}

The SIR of the D2D system at the output of $D$ branches MRC combiner is

$$
\frac{S_{S, M R C}}{S_{I}}=\frac{P_{1}\left(\frac{x_{0}{ }^{u-2}}{x^{u}}\right) \sum_{d=1}^{D} h_{d}}{\sum_{n=1}^{N} P_{I, n}\left(\frac{y_{0, n}^{v_{n}-2}}{y_{n}^{v_{n}}}\right) \beta_{n}} .
$$

where $S_{S, M R C}$ is the received power of the D2D signal. The outage probability for the MRC based D2D system is

$$
P_{o u t, M R C}=\operatorname{Pr}\left(R S_{I}>S_{S, M R C}\right)
$$

Using the expression given in (11), a decision variable $\theta$ is defined as

$$
\theta=R S_{I}-S_{S, M R C}
$$

For a satisfactory desired D2D signal quality, the value of $\theta$ has to be negative. Otherwise, outage will happen. Mathematically,

$\theta \begin{cases}>0 & \text { Outage } \\ \leq 0 & \text { Acceptable Transmission }\end{cases}$

The CF of the decision variable $\theta$ is given as

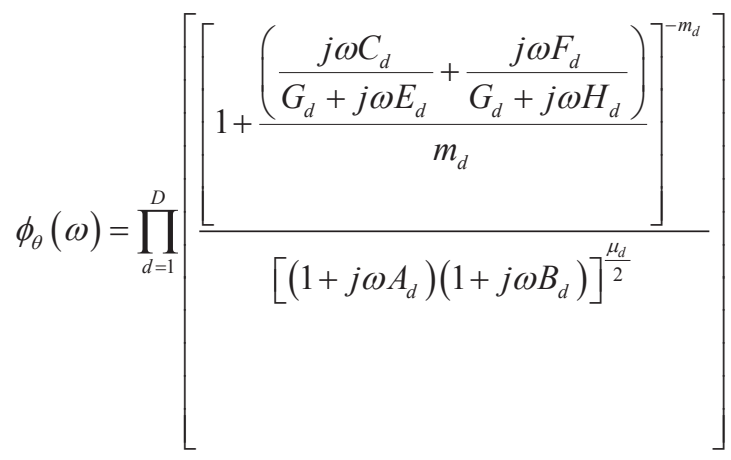


Performance Analysis of Communication System

with Fluctuating Beckmann Fading

$$
\times \prod_{n=1}^{N}\left[\frac{\left[1-\frac{\left(\frac{j \omega C_{n}}{G_{n}-j \omega E_{n}}+\frac{j \omega F_{n}}{G_{n}-j \omega H_{n}}\right)}{m_{n}}\right]^{-m_{n}}}{\left[\left(1-j \omega A_{n}\right)\left(1-j \omega B_{n}\right)\right]^{\frac{\mu_{n}}{2}}}\right]
$$

Based on (13) and (14), the outage probability expression for independent but non-identically distributed D2D and CCI signals is

$$
P_{\text {out }, \text { MRC }}=\frac{1}{2}+\frac{1}{\pi} \int_{0}^{\infty} \frac{\operatorname{Im}\left(\phi_{\theta}(\omega)\right)}{\omega} d \omega
$$

The outage probability expression for independent and identically distributed system is

$$
P_{\text {out }, M R C}=\frac{1}{2}+\frac{1}{\pi} \int_{0}^{\infty} \frac{\operatorname{Im}\left(\left[X_{d}\right]^{D}\left[Y_{n}\right]^{N}\right)}{\omega} d \omega
$$

where

and

$$
Y_{n}=\frac{\left[1-\frac{1}{m_{n}}\left(\frac{j \omega C_{n}}{G_{n}-j \omega E_{n}}+\frac{j \omega F_{n}}{G_{n}-j \omega H_{n}}\right)\right]^{-m_{n}}}{\left(1-j \omega A_{n}\right)^{\frac{\mu_{n}}{2}}\left(1-j \omega B_{n}\right)^{\frac{\mu_{n}}{2}}}
$$

The success probability expression for the independent and non-identically distributed system is

$$
P_{S, M R C}=\frac{1}{2}-\frac{1}{\pi} \int_{0}^{\infty} \frac{\operatorname{Im}\left(\phi_{\theta}(\omega)\right)}{\omega} d \omega
$$

The success probability expression for the independent and identically distributed case is

$$
P_{S, M R C}=\frac{1}{2}-\frac{1}{\pi} \int_{0}^{\infty} \frac{\operatorname{Im}\left(\left[X_{d}\right]^{D}\left[Y_{n}\right]^{N}\right)}{\omega} d \omega
$$

\section{NUMERICAL RESULTS AND ANALYSIS}

In this section, the performance of FB faded D2D system based on the expressions derived in Section II is presented. The reference distances $x_{0}$ and $y_{0, n}$ are assumed to be 1 meter. Fig. 2 shows the outage performance of D2D system with the various number of diversity branches for both $\mathrm{SC}$ and MRC schemes. The values for D2D signals parameters for number of diversity branches $D=2$ and 3, and CCI signals parameters are shown in Table 1 .

\begin{tabular}{|l|l|l|l|}
\hline \multicolumn{4}{|c|}{ Table 1. } \\
\hline Parameters & Values & Parameters & Values \\
\hline$u$ & 3.4 & $P_{I, n}$ & $\begin{array}{l}{[16.98,17.78,18.45,} \\
19.03,20] \mathrm{dBm}\end{array}$ \\
\hline$x$ & 15 meters & $v_{n}$ & $\begin{array}{l}{[2.7,2.8,2.9,3.0,} \\
2.6]\end{array}$ \\
\hline$\mu_{d}$ & {$[10,7] \&[10,7,1]$} & $y_{n}$ & $\begin{array}{l}{[45,40,35,30,25]} \\
\text { meters }\end{array}$ \\
\hline$\kappa_{d}$ & {$[10,3] \&[10,3,8]$} & $\mu_{n}$ & {$[1,3,7,3,4]$} \\
\hline$\zeta_{d}{ }^{2}$ & {$[0.1,0.01] \&[0.1$,} & $\zeta_{n}{ }^{2}$ & {$[0.01,0.1,0.001,1$,} \\
\hline$\eta_{d}$ & $0.01,1]$ & $2]$ \\
\hline$m_{d}$ & {$[10,5] \&[10,5,6]$} & $\kappa_{n}$ & {$[1,5,6,4,5]$} \\
\hline$R$ & {$[3,2] \&[3,2,7]$} & $\eta_{n}$ & {$[1,2,7,2,3]$} \\
\hline
\end{tabular}

From the figure, it is clear that as the number of branches is increased for the SC and MRC schemes outage performance improves. It is because of the improved SIR conditions of the system. Furthermore, it is seen that the increase in power of the D2D signal improves the outage performance of the system. Analytical and simulation of Outage performance of D2D communication system with various values $\mu_{d}$ and $x$ is shown in Fig. 3. The values for D2D signals parameters and CCI signals parameters are shown in Table 2.

\begin{tabular}{|l|l|l|l|}
\multicolumn{4}{|c|}{ Table 2.} \\
\begin{tabular}{|l|l|l|l|}
\hline Parameters & Values & Parameters & Values \\
\hline$P_{1}$ & $20 \mathrm{dBm}$ & $v_{n}$ & {$[3,3.1,3.2,3.3,3]$} \\
\hline$u$ & 2.6 & $\mu_{n}$ & {$[1,3,7,3,4]$} \\
\hline$\kappa_{d}$ & {$[0,0]$} & $\kappa_{n}$ & {$[0,0,0,0,0]$} \\
\hline$\eta_{d}$ & {$[0,0]$} & $\eta_{n}$ & {$[0,0,0,0,0]$} \\
\hline$m_{d}$ & {$[0,0]$} & $m_{n}$ & {$[0,0,0,0,0]$} \\
\hline$\zeta_{d}{ }^{2}$ & {$[0,0]$} & $\zeta_{n}{ }^{2}$ & {$[0,0,0,0,0]$} \\
\hline$P_{I, n}$ & {$[20,23.01,24.77$,} & $y_{n}$ & {$[45,40,35,30,30]$} \\
& $26.02,26.99] \mathrm{dBm}$ & & meters \\
\hline
\end{tabular}
\end{tabular}

From the figure, it is observed that the outage probability of the system is less for higher values fading parameter $\mu_{d}$. It is because of the fading condition of D2D signals which results in an improved outage performance of the D2D system. Moreover, the increase in distance between D2D devices degrades the outage performance of the system. It is because of the path-loss phenomena. Outage performance of D2D system for various values of $v_{n}$ and $x$ is shown in Fig. 4. The values for the three branches of MRC based D2D signal parameters and CCI signal Parameters are given in Table 3. 
Table 3.

\begin{tabular}{|l|l|l|l|}
\hline Parameters & Values & Parameters & Values \\
\hline$P_{1}$ & $20 \mathrm{dBm}$ & $\mathrm{P}_{I, n}$ & $\begin{array}{l}{[16.98,17.78,18.45,} \\
19.03,20] \mathrm{dBm}\end{array}$ \\
\hline$u$ & 3.2 & $\mu_{n}$ & {$[1,3,4,3,2]$} \\
\hline$\kappa_{d}$ & {$[10,3,8]$} & $\kappa_{n}$ & {$[1,2,3,4,1]$} \\
\hline$\eta_{d}$ & {$[10,5,6]$} & $\eta_{n}$ & {$[1,2,3,2,3]$} \\
\hline$m_{d}$ & {$[6,5,7]$} & $m_{n}$ & {$[1,3,4,2,3]$} \\
\hline$\zeta_{d}^{2}$ & {$[0.1,0.01,1]$} & $\zeta_{n}^{2}$ & {$[0.01,0.1,0.001,0.5,1]$} \\
\hline$\mu_{d}$ & {$[10,7,1]$} & $y_{n}$ & $\begin{array}{l}{[25,30,35,40,45]} \\
\text { meters }\end{array}$ \\
\hline
\end{tabular}

From the figure, it is observed that the outage probability of the system is less for higher values CCI path-loss exponents. It is because of the weakening of CCI signals which results in an improved outage performance of the D2D system. Moreover, the increase in distance between D2D devices degrades the outage performance of the system.

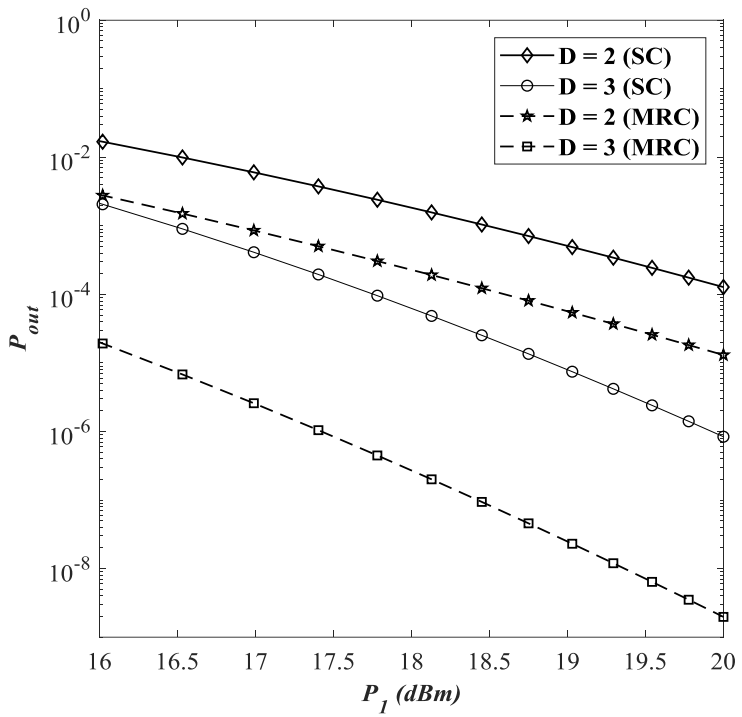

Fig. 2. Outage probability for various numbers of SC and MRC branches

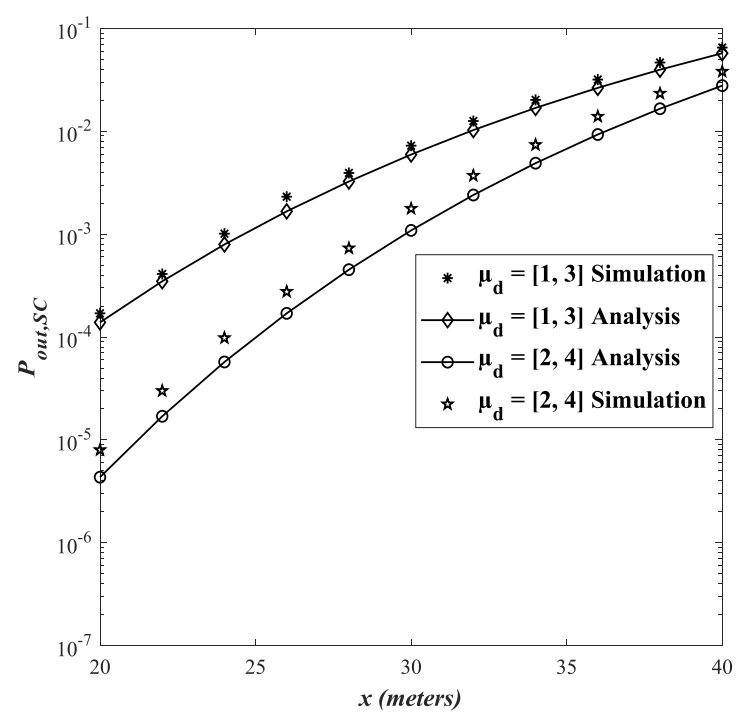

Fig. 3. Outage Probability for various values of fading parameter
In Fig. 5, outage performance of D2D system for various values of $u$ and $m_{d}$ is shown. The values for the three branches of SC based D2D signal parameters and CCI signal parameters are given in Table 4.

Table 4.

\begin{tabular}{|l|l|l|l|}
\hline Parameters & Values & Parameters & Values \\
\hline$P_{1}$ & $20 \mathrm{dBm}$ & $\mathrm{P}_{I n}$ & $\begin{array}{l}{[16.98,17.78,18.45,} \\
19.03,20] \mathrm{dBm}\end{array}$ \\
\hline$x$ & 19 meters & $\mu_{n}$ & {$[1,3,7,3,4]$} \\
\hline$\kappa_{d}$ & {$[10,3,8]$} & $\kappa_{n}$ & {$[1,5,6,4,5]$} \\
\hline$\eta_{d}$ & {$[10,5,6]$} & $\eta_{n}$ & {$[1,2,7,2,3]$} \\
\hline$\mu_{d}$ & {$[10,7,1]$} & $m_{n}$ & {$[1,3,4,2,3]$} \\
\hline$\zeta_{d}{ }^{2}$ & {$[0.1,0.01,1]$} & $\zeta_{n}{ }^{2}$ & $\{0.01,0.1,0.001,1,2\}$ \\
\hline$v_{n}$ & {$[2.7,2.8,2.9,3$,} & $y_{n}$ & $\begin{array}{l}{[25,30,35,40,45]} \\
\text { meters }\end{array}$ \\
\hline
\end{tabular}

From the figure, it is observed that the outage probability of the system is worse for the higher values of the $u$. It is due to the weakened D2D signal due to the path-loss effects. Moreover, from the figure it can also be seen that the outage performance of the system improves as the values of $m_{d}$ is increased. It is because of the better shadowing conditions of the D2D communication channel.

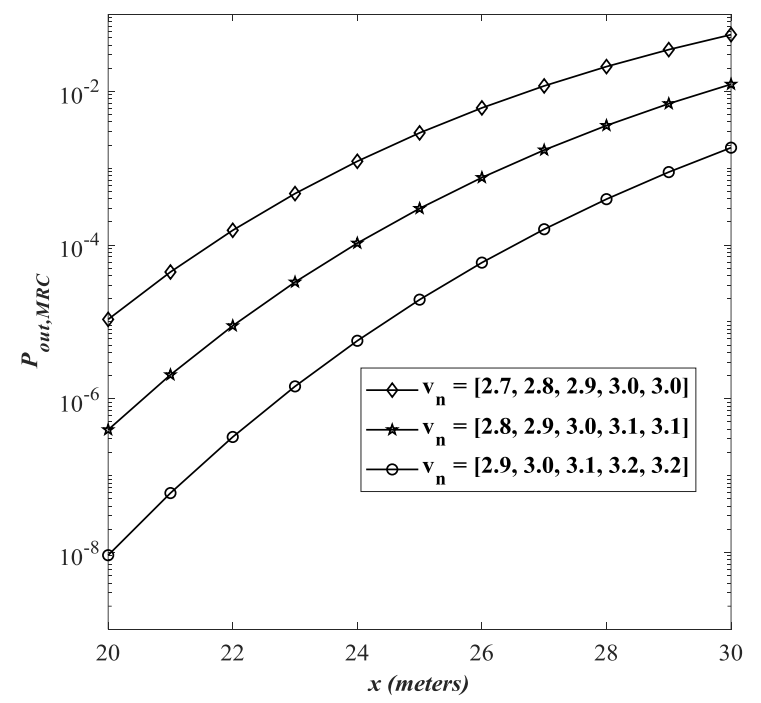

Fig. 4. Outage performance of MRC based D2D system with varying pathloss exponents of CCI signals

Fig. 6 presents outage performance of D2D communication system with varying values of $m_{n}$. The values for $P_{1}, P_{I, n}, x, y_{n}$, $u, v_{n}, \mu_{d}, \mu_{n}, \kappa_{d}, \kappa_{n}, \eta_{d}, \eta_{n}, \zeta_{d}{ }^{2}, \zeta_{n}{ }^{2}$ and $R$ are considered to be 20 $\mathrm{dBm}, 16.98 \mathrm{dBm}, 15$ meters, 35 meters, 3.3, 2.7, 2, 2, 5, 4, 0.1, $0.1,0.01,0.01$ and $20 \mathrm{dBm}$, respectively. From the figure it is observed that the outage performance is almost insensitive to the variations of $m_{n}$. Furthermore, it is also observed that the outage performance improves as the values of $m_{d}$ is increased. 
Performance Analysis of Communication System

with Fluctuating Beckmann Fading

Outage performance of D2D system for the varying values of $\zeta_{n}{ }^{2}$ and $\eta_{n}$ in a scenario with $\mu_{n}=1$ and $\mu_{d}$ is shown in Fig. 7. The values for $P_{1}, P_{I, n}, x, y_{n}, u, v_{n}, \kappa_{d}, \kappa_{n}, \eta_{d}, m_{d}, m_{n}, \zeta_{d}^{2}$ and $R$ are set to be $20 \mathrm{dBm}, 16.98 \mathrm{dBm}, 15$ meters, 40 meters, 3.5, 2.5, $10,1,10,3,5,0.1$, and $16.98 \mathrm{dBm}$, respectively. From the figure it is observed that outage performance of the system is better for the higher values of $\eta_{n}$. Moreover, from the figure it can be seen that the outage performance improves as the number of clusters of desired D2D signal increases.

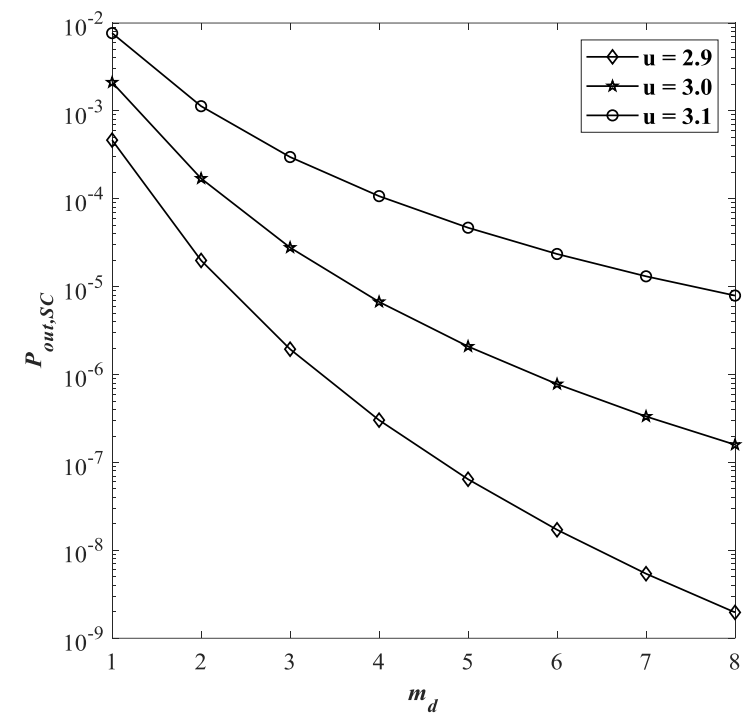

Fig. 5. Outage performance of SC based D2D system for various values of path-loss exponent $u$

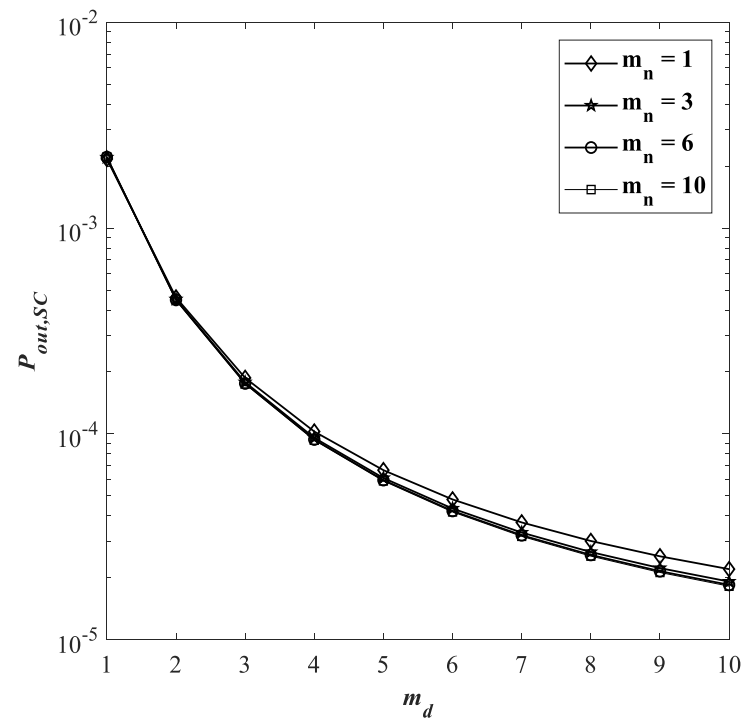

Fig. 6. Outage performance of SC based D2D system for various values of $m_{n}$

Success probability performance of D2D system for $\kappa_{n}$ and $\kappa_{d}$ is shown in Fig. 8. The values for $P_{1}, P_{I, n}, x, y_{n}, u, v_{n}, \eta_{d}, \eta_{n}, m_{d}$, $m_{n}, \mu_{d}, \mu_{n}, \zeta_{d}{ }^{2}, \zeta_{n}{ }^{2}$ and $R$ are set to be $20 \mathrm{dBm}, 16.98 \mathrm{dBm}, 15$ meters, 30 meters, 3.8, 2.5, 2, 2, 3, 1, 2, 2, 0.01, 0.01 and 16.98
$\mathrm{dBm}$, respectively. From the figure it is observed that success probability performance of the system is better for the lower values $\kappa_{n}$. It is because of degraded CCI signals which results in an improved the SIR performance of the system, and hence better success probability performance of the system. It can also be seen that the success probability of the system increases as the values of $\kappa_{d}$ increases. In Fig. 9, success probability performance of D2D communication system with varying values of and is shown. $P_{1}, P_{I, n}, x, y_{n}, u, v_{n}, \kappa_{d}, \kappa_{n}, \eta_{d}, \eta_{n}, m_{d}$, $m_{n}, \mu_{d}, \mu_{n}$ and $R$ are fixed at $20 \mathrm{dBm}, 16.98 \mathrm{dBm}, 16$ meters, 25 meters, $3.5,2.5,1,5,10,2,1,1,3,3$ and $20 \mathrm{dBm}$, respectively. From the figure, it can be seen that the success probability does not show much variation when $\zeta_{n}^{2}$ is varied. Furthermore, it is also observed that the success probability performance of the system deteriorates as $\zeta_{d}^{2}$ is increased.

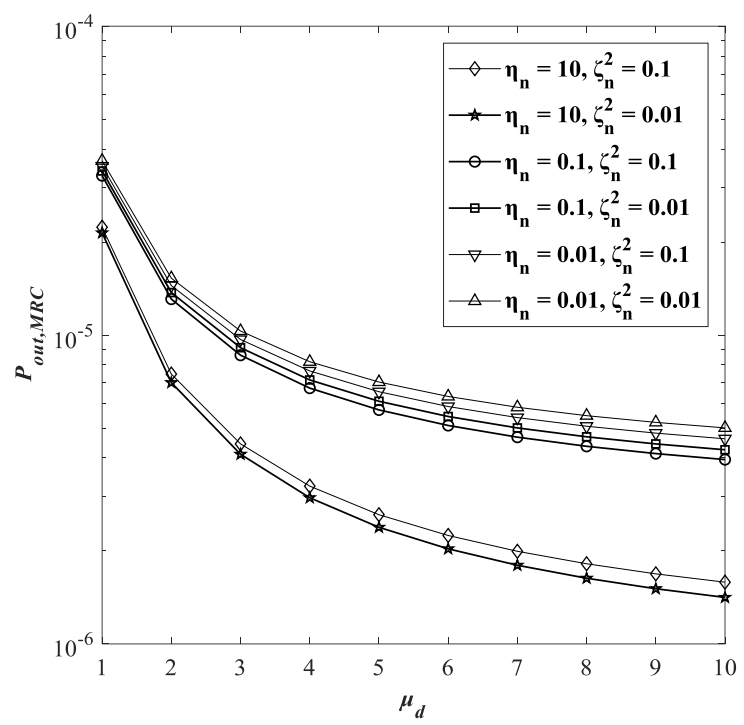

Fig. 7. Outage performance of MRC based D2D system for various values of $\eta_{n}$ and $\zeta_{n}^{2}$

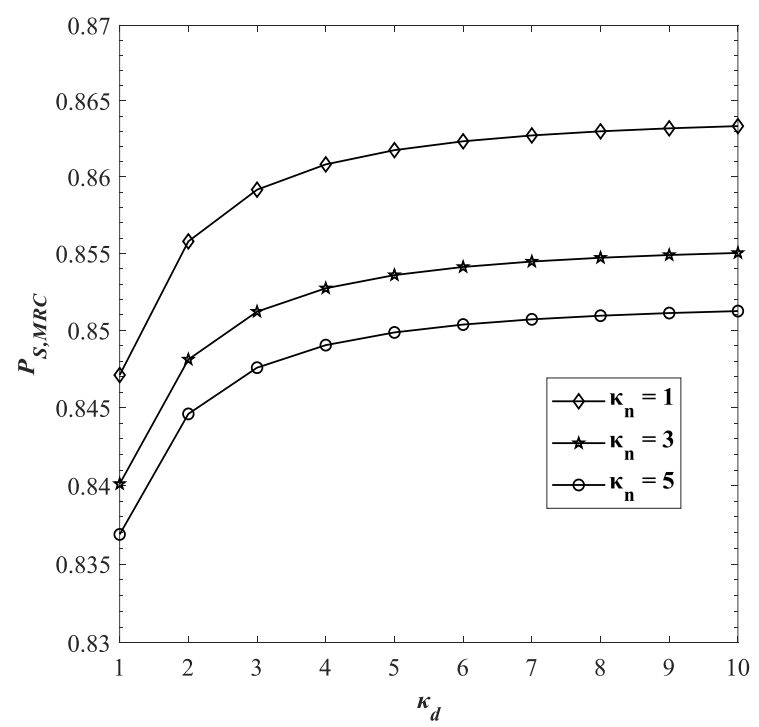

Fig. 8. Success probability performance of MRC based D2D system for various $\kappa_{n}$ 
Success probability performance of the D2D system with varying values $\mu_{n}$ and the distance $y$ is analyzed in Fig. 10. Here, the values for parameters $P_{1}, P_{I, n}, x, u, v_{n}, \kappa_{d}, \kappa_{n}, \eta_{d}, \eta_{n}, m_{d}, m_{n}$, $\zeta_{d}^{2}, \zeta_{n}^{2}$ and $\mu_{n}$ are fixed at $20 \mathrm{dBm}, 17.78 \mathrm{dBm}, 16$ meters, 3.5, $2.5,1,5,10,5,1,10,10,0.1$ and 2 , respectively. From the figure, it is evident that the success probability performance of the system is almost insensitive to the change in the values of $\mu_{n}$. Moreover, from the figure it is also seen that the success probability of the system improves as the distance $y$ is increased. It is because of the improved SIR conditions of the system due to weakening of CCI signals by the path-loss.

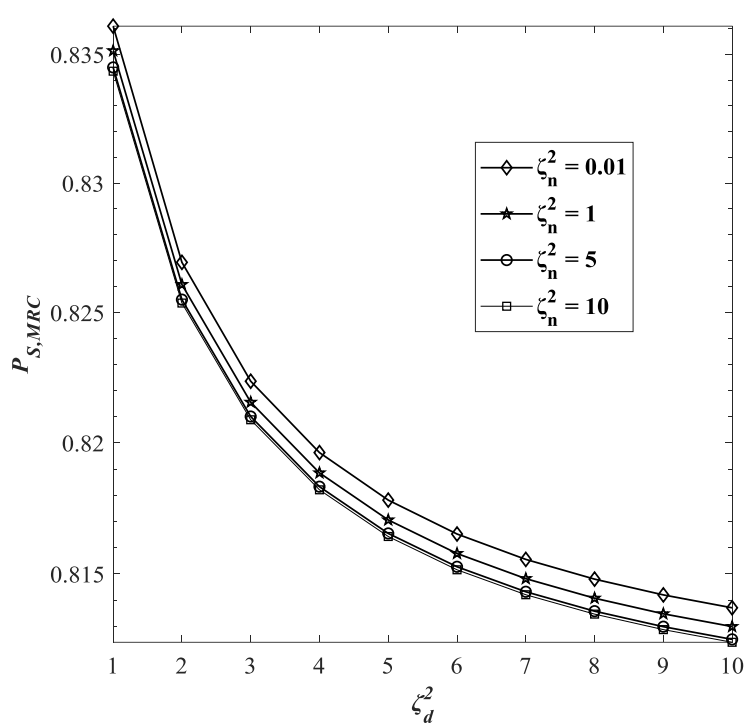

Fig. 9. Success probability performance of MRC based D2D system with varying values of $\zeta_{n}^{2}$

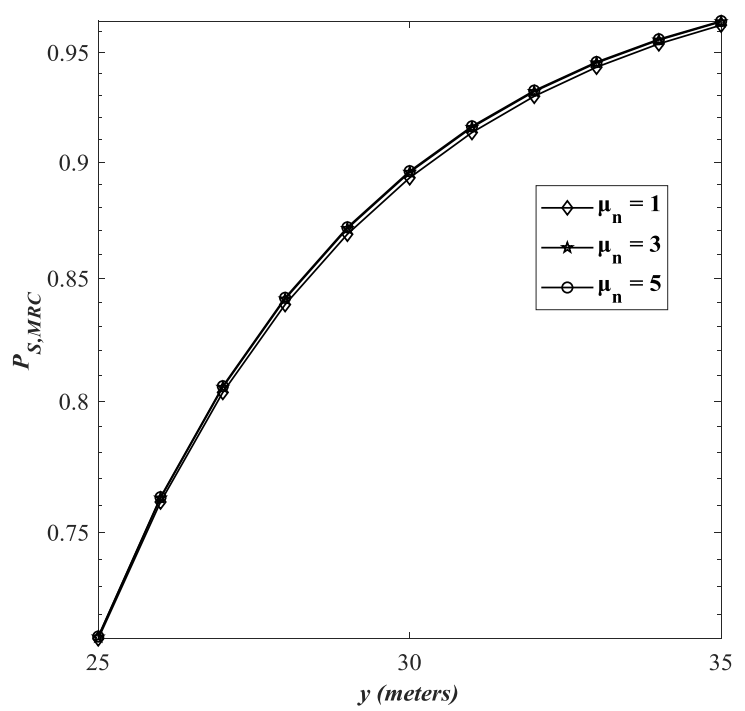

Fig. 10. Success Probability of MRC based D2D system with varying values of $\mu_{n}$

\section{CONCLUSION}

In this paper, outage and success performances of a D2D communication system over a Fluctuating Beckmann (FB) fading channel in an interference limited scenario is analyzed. The FB distribution generalizes various distributions. Expressions of outage and success probabilities using characteristic function (CF) based approach is presented. Effects of co-channel interference, FB channel conditions and the path-loss on the outage and success probabilities of the system are presented and discussed. MRC and SC diversity schemes are also incorporated to mitigate fading conditions. It is observed that path-loss significantly effects performance of D2D system. It is also observed that the system performance improves as the number of clusters in the D2D signal is increased. However, system performance is almost insensitive to the variations in the number of clusters of CCI.

\section{APPENDIX A}

ProOF OF EQ. No. 5

$$
\begin{aligned}
\partial=R S_{I}-S_{S, S C, M A X} \\
\phi_{\partial}(\omega)=E\left(e^{j \omega\left(R S_{I}-S_{S, S C, M A X}\right)}\right) \\
=E\left(e^{j \omega\left(R S_{I}\right)} e^{j \omega\left(-S_{S, S C, M A X}\right)}\right) \\
=E\left(e^{j \omega\left(\sum_{n=1}^{N} \delta_{n} \beta_{n}\right)} e^{j \omega\left(-\delta_{d} h_{d}\right)}\right) \\
=E\left(e^{j \omega\left(\sum_{n=1}^{N} \delta_{n} \beta_{n}\right)}\right)^{E}\left(e^{j \omega\left(-\delta_{d} h_{d}\right)}\right) \\
=E\left(e^{j \omega\left(-\delta_{d} h_{d}\right)}\right) \prod_{n=1}^{N} E\left(e^{j \omega \delta_{n} \beta_{n}}\right) \\
=\phi_{d}\left(-\omega \delta_{d} h_{d}\right) \prod_{n=1}^{N} \phi_{I}\left(\omega \delta_{n} \beta_{n}\right)
\end{aligned}
$$

\section{REFERENCES}

[1] You, D. and Dong H. K., "Hybrid STBC-SM Suitable for Multi-link Device-to-Device Communication in Cellular Networks," Wireless Personal Communications, vol. 96, no. 1, pp. 1507-1518., Sept. 2017, [https://doi.org/10.1007/s11277-017-4253-9].

[2] Sharifi, S. and Mohammad F., "Underlay device to device communication with imperfect interference channel knowledge," Wireless Personal Communications, vol. 101, no. 2, pp. 619-634, July 2018, [https://doi.org/10.1007/s11277-018-5707-4]. 
[3] Schreck, J., Jung P. and Stańczak, S., "Compressive Rate Estimation With Applications to Device-to-Device Communications," in IEEE Transactions on Wireless Communications, vol. 17, no. 10, pp. 70017012, Oct. 2018, [https://doi.org/10.1109/TWC.2018.2865347].

[4] Wang, J., Huang, Y., Jin, S., Schober, R., You X. and Zhao, C., "Resource Management for Device-to-Device Communication: A Physical Layer Security Perspective," in IEEE Journal on Selected Areas in Communications, vol. 36, no. 4, pp. 946-960, April 2018, [https://doi.org/10.1109/JSAC.2018.2825484].

[5] Liu, F., Hou, X. and Liu, Y., "Capacity Improvement for Full Duplex Device-to-Device Communications Underlaying Cellular Networks," in IEEE Access, vol. 6, pp. 68373-68383, 2018, [https://doi.org/10.1109/ACCESS.2018.2879472].

[6] Huq, K. M. S., Mumtaz, S. and Rodriguez, J., "Outage probability analysis for device-to-device system," 2016 IEEE International Conference on Communications (ICC), Kuala Lumpur, 2016, pp. 1-5, [https://doi.org/10.1109/ICC.2016.7510678].

[7] Khuntia, P. and Hazra, R., "Resource sharing for device-to-device communication underlaying cellular network," 2018 4th International Conference on Recent Advances in Information Technology (RAIT), Dhanbad, 2018, pp. 1-5 [https://doi.org/10.1109/RAIT.2018.8389093].

[8] Liu, J., Nishiyama, H., Kato, N. and Guo, J., "On the Outage Probability of Device-to-Device-Communication-Enabled Multichannel Cellular Networks: An RSS-Threshold-Based Perspective," in IEEE Journal on Selected Areas in Communications, vol. 34, no. 1, pp. 163-175, Jan. 2016, [https://doi.org/10.1109/JSAC.2015.2452492].

[9] Peng, M., Li, Y., Quek, T. Q. S. and Wang, C., "Device-to-Device Underlaid Cellular Networks under Rician Fading Channels," in IEEE Transactions on Wireless Communications, vol. 13, no. 8, pp. 42474259, Aug. 2014, [https://doi.org/10.1109/TWC.2014.2314115].

[10] Ramirez-Espinosa, P., Lopez-Martinez, F. J., Paris, J. F. , Yacoub, M. D. and Martos-Naya, E., "An Extension of the $\varkappa-\mu$ Shadowed Fading Model: Statistical Characterization and Applications," in IEEE Transactions on Vehicular Technology, vol. 67, no. 5, pp. 3826-3837, May 2018 [https://doi.org/10.1109/TVT.2017.2787204].

[11] J.F. Paris, "Statistical Characterization of $x-\mu$ Shadowed Fading,", in IEEE Transactions on Vehicular Technology, vol. 63, no. 2, pp. 518-526, Feb. 2014, [https://doi.org/10.1109/TVT.2013.2281213].

[12] Goldsmith, A., Wireless communications, Cambridge university press, 2005

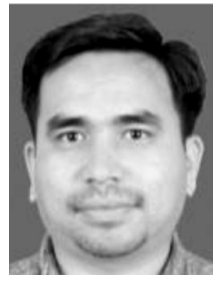

Zakir Hussain received M.S. degree in Electrical Engineering from National University of Computer and Emerging Sciences (NUCES), Karachi Campus in 2017. Research areas includes Device-to-Device communication, interference analysis in wireless communication systems, digital communication systems, signal processing.

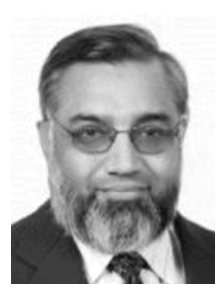

Asim ur Rehman Khan received BSc degree in Electrical Engineering (EE) from UET Lahore, Pakistan in 1981, MS EE from South Dakota State University, South Dakota, USA in 1987, and PhD EE from Polytechnic University (now NYU) in Brooklyn, New York, 8 USA in 1993. His main area of research is AI, Computer Vision, and Pattern Recognition. He has worked in various telecom projects in Pakistan. Since 2002, he is Professor of Electrical Engineering at the Karachi campus of National University of Computer; Emerging Sciences (NUCES), Pakistan.

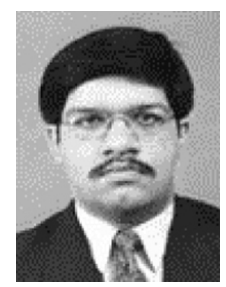

Haider Mehdi research interests include Deviceto-Device communication, interference analysis in wireless communication systems, digital communication systems, digital signal processing, and signal-processing techniques for the communication systems.

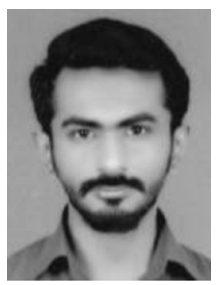

Aamir Ali received the B.S. and M.S. degrees in Electrical Engineering from National University of Computer and Emerging Sciences in 2015 and 2019, respectively. $\mathrm{He}$ has been associated with the faculty of Electrical Engineering Department at National University of Computer and Emerging Sciences since Jan 2016. He has been author of many research papers. His research interest include Robotics and Telecommunication. 